\title{
Hiperoxaluria primaria tipo 1
}

\section{Primary hyperoxaluria type 1}

\author{
luisa Patiño, Julián Rondón, Catalina Hernández \\ - Bogotá, D.C. (Colombia)
}

\section{Introducción}

Se presenta el caso de una paciente con historia personal de urolitiasis a repetición desde su infancia, con deterioro progresivo de la función renal hasta el punto de requerir terapia de reemplazo renal dado extenso compromiso por nefrocalcinosis en la segunda década de la vida. Unos años después, partiendo de un análisis detallado de sus múltiples manifestaciones clínicas, se logró llegar a un diagnóstico retrospectivo de hiperoxaluria primaria tipo 1, un raro trastorno genético que es causa no sólo de insuficiencia renal avanzada de inicio precoz, sino también de un espectro clínico mucho más amplio que el comprendido dentro del grupo de trastornos del metabolismo

El objetivo al compartir el caso, es sensibilizar al clínico en las indicaciones para el estudio precoz de pacientes con urolitiasis, con el fin de impactar decididamente en la historia natural de este tipo de trastornos enzimáticos y mejorar pronóstico y calidad de vida de este tipo de pacientes.

\section{Presentación del caso}

Mujer de 37 años con antecedente de enfermedad renal crónica terminal, en terapia de reemplazo renal (hemodiálisis) desde hace 16 años, actualmente en plan de trasplante renal de donante vivo, quien consulta por disfunción de fístula arteriovenosa (FAV) braquial derecha. Además de la historia de trombosis crónica con recanalización tardía de sus accesos vasculares, llamaba la atención la historia de uropatía obstructiva desde los seis años, livedo reticularis, múltiples lesiones de aspecto vasculítico de predominio en extremidades, antecedente de fracturas por fragilidad ósea e historia familiar de enfermedad renal crónica avanzada antes de la segunda década en hermana, quien había fallecido al parecer por evento coronario a temprana edad. Refería además dolor de características neuropáticas en miembros inferiores. A pesar de que muchos de estos hallazgos clínicos podrían estar explicados por trastornos del metabolismo mineral óseo dentro del espectro de enfermedad renal crónica, se decidió avanzar en estudios para descartar causas secundarias que explicaran la presentación atípica y precoz de estas manifestaciones.

De esta manera, se realizó una serie radiográfica de tórax, abdomen y pelvis, que mostró lesiones líticas y blásticas, sin presencia de quistes subcondrales o geodas. Se complementó este estudio con gamagrafía ósea, que reportó hipercaptación difusa en columna vertebral y articulaciones sacroiliacas, como hallazgo sugestivo de trastorno metabólico por depósito. En este punto, se solicitaron niveles de calcio, fósforo, 25-hidroxivitamina D y PTH, que descartaron hiperparatiroidismo secundario, terciario u osteomalacia por deficiencia severa en niveles de vitamina D. Por otro lado, y ante sospecha de calcifilaxis o un proceso vasculítico secundario, se hizo una biopsia de piel que reportó extensos depósitos de cristales de oxalato en tejidos blandos y espacios perivasculares. Se solicitaron estudios para causas secundarias de hiperoxalosis, los cuales fueron negativos. Ante la alta sospecha clínica de trastorno primario en el metabolismo del oxalato por deficiencia enzimática específica, se ordenó estudio genético para la mutación del gen AGXT, el cual fue positivo, confirmándose de esta manera la entidad.
Dra. Luisa Patiño U., Dr. Julián Rondón C.: Residentes de Medicina Interna: Dra. Catalina Hernández F.: Profesora Asistente. Pontificia Universidad Javeriana, Departamento de Medicina Interna, Hospital Universitario San Ignacio. Bogotá, D.C. (Colombia).

Correspondencia: Dra. Luisa Patiño U. Bogotá, D.C. (Colombia).

E-mail: luisaf.2301@hotmail.com 


\section{Discusión}

Las hiperoxalurias tipo I, II y III son trastornos heredados de manera autosómica recesiva, causados por defectos en el metabolismo del glioxilato, que llevan a la sobreproducción de oxalato. El reconocimiento inicial de estos trastornos metabólicos se atribuye a Lepoutre, quien reportó los primeros casos en 1925, lográndose describir los procesos bioquímicos involucrados varios años después $(1,2)$.

Aunque se clasifican según la enzima deficiente dentro del circuito de peroxisomas hepáticos, todas las formas se caracterizan por una excreción urinaria de oxalato aumentada (>1 mmol/1.73 m2 SC/día), lo cual resulta en urolitiasis recurrente y/o nefrocalcinosis progresiva $(1,4)$. A continuación, el deterioro de la función renal lleva a disminución en la depuración de oxalato, lo cual perpetúa la acumulación sistémica de cristales de oxalato de calcio. Aunque las manifestaciones clínicas son prevenibles con un diagnóstico precoz, la mayoría de veces el diagnóstico es tardío y se realiza en pacientes con enfermedad renal crónica terminal establecida, lo cual ocurre en más del $30 \%$ de pacientes con hiperoxaluria primaria tipo 1 $(2,3)$, donde el aumento en la producción hepática de oxalato supera los mecanismos de eliminación del mismo, llevando a toxicidad directa, nefrocalcinosis (depósitos de cristales a nivel intratubular e intersticial) y posterior obstrucción renal por litos, que usualmente se asocian a infecciones de las vías urinarias. Esta saturación de los mecanismos fisiológicos de depuración del oxalato se alcanza cuando la tasa de filtración glomerular (TFG) cae a 30-45 ml/min/1.73 $\mathrm{m}^{2} \mathrm{SC}$, lo cual coincide con el depósito subclínico de oxalato en la matriz ósea y posteriormente, en tejido celular subcutáneo u órganos sólidos, fenómeno conocido como la oxalosis sistémica.

El oxalato es un ácido dicarboxílico (HOOC-COOH) altamente insoluble, producto final del metabolismo en los seres humanos (2-4). Se excreta casi por completo por vía renal (en forma de sal cálcica), con una tendencia conocida a depositarse en los túbulos renales. Además de los defectos enzimáticos ya mencionados, existen causas secundarias de hiperoxalosis como resultado del exceso en la ingesta dietaria de precursores del oxalato (espinaca, remolacha) o por hiperoxaluria entérica, esto es, en pacientes con antecedente de resección intestinal o enfermedad inflamatoria intestinal, en quienes se aumenta el secuestro intestinal de calcio por parte del colon, incluyendo así las formas más solubles de oxalato. Estas situaciones se deben descartar primero antes de considerar diagnóstico de hiperoxaluria primaria $(6,7)$.

La hiperoxaluria primaria es considerada una enfermedad huérfana $(2,5,6)$. Aunque se desconoce la prevalencia real, la forma más común (tipo 1) tiene una prevalencia estimada de uno a tres casos por millón de personas y una incidencia de aproximadamente un caso por 120000 nacidos vivos por año en Europa. Así mismo, en los lugares de mayores reportes de la entidad (Europa, Japón, EE.UU.), se considera que representa entre 1 y $2 \%$ de las causas de enfermedad renal crónica terminal en población pediátrica. No hay datos locales en Colombia que permitan una aproximación en términos epidemiológicos.
En este caso, a diferencia de los tipos II y III, se ha descrito un trastorno en el metabolismo del glioxilato debido a una alteración constitutiva de la enzima hepática glioxilatoaminotransferasa peroxisomal, lo cual lleva a un aumento en la síntesis de ácido glicólico y oxálico, con posterior sobreproducción de oxalato y todas las implicaciones clínicas descritas, secundarias al depósito sistémico $(1,2,4)$.

El diagnóstico se puede establecer mediante la determinación de oxalato, glicolato o glicerato en orina, aunque a la fecha existen pruebas moleculares y genéticas orientadas hacia las mutaciones ya conocidas. Finalmente, es posible la cuantificación enzimática en el parénquima hepático, además de la detección de cristales de oxalato en el mismo por tejido extraído por biopsia.

Las opciones de tratamiento incluyen citrato alcalino, ortofosfato o magnesio, conocidos como inhibidores de la cristalización de los cristales de oxalato $(1,5-7)$. Así mismo, la terapia con piridoxina ha demostrado normalizar o reducir la excreción de oxalato hasta en un tercio de pacientes con HP tipo 1. La terapia dialítica no evita la acumulación sistémica de depósitos de oxalato. Las opciones definitivas de tratamiento están orientadas al trasplante de órgano sólido (hígado, riñón) y van a depender del tipo de enzima afectada y de cada caso en particular. En HP tipo 1, la elección es el trasplante combinado hígado/riñón a edades tempranas, mientras que el trasplante renal aislado se prefiere en los casos de HP tipo 2.

En el futuro se han planteado distintas estrategias, como el trasplante de hepatocitos, la terapia génica de reemplazo enzimático mediada por microorganismos vivos como adenovirus, entre otras técnicas aún en estudio.

\section{Referencias}

1. Hoppe, Bernd. An update on primary hyperoxaluria. University Hospital, Department of Pediatric and Adolescent Medicine, Division of Pediatric Nephrology, Kerpener Strasse 62, D50931 Cologne, Germany. bernd.hoppe@ uk-koeln.de. Nature reviews I Nephrology volume 8 I august 2012. URL: http://www.nature. $\mathrm{com} / \mathrm{nrneph} /$ journal/v8/n8/full/nrneph.2012.113.html?foxtrotcallback=true

2. Cochat, Pierre et al. Primary hyperoxaluria. Centre de Référence des Maladies Rénales Rares Néphrogones; Centre de la Recherche Scientifique - Unité Mixte de la Recherche 5305, Hospices Civils de Lyon, and Université Claude-Bernard Lyon. N Engl J Med. 2013; 369: 649-58. URL: http://www.nejm.org/doi/full/10.1056/ NEJMra1301564.

3. Bashin, Bhavna et al. Primary and secondary hyperoxaluria: Understanding the enigma. Division of Nephrology, Medical University of South Carolina, Charleston, SC 29425, United States. World J Nephrol. 2015 May 6; 4 (2): 235-244. URL: https:/www.ncbi.nlm.nih.gov/pmc/articles/PMC4419133/

4. Leal, Rita et al. Primary hyperoxaluria type 1:A literature review upon three clinical cases. Department of Nephrology, Centro Hospitalar e Universitário de Coimbra. Coimbra, Portugal. Port J Nephrol Hypert 2014; 28 (4): 281-289. URL: http://www. scielo.mec.pt/pdf/nep/v28n4/28n4a02.pdf

5. Spasovski, Goce et al. Late diagnosis of primary hyperoxaluria after failed kidney transplantation. University Department of Nephrology, Medical Faculty, University of Skopje, Vodnjanska 17, 1000 Skopje,Macedonia. Nephrology: case report. Int Urol Nephrol. URL: http://www.biopku.org/pdf/spasovski_2009_iun.pdf

6. Cochat, Pierre et al. Primary hyperoxaluria Type 1: indications for screening and guidance for diagnosis and treatment. Nephrol Dial Transplant (2012) 27: 1729-1736 doi: 10.1093/ndt/gfs078. URL: https://academic.oup.com/ndt/articlelookup/doi/10.1093/ndt/gfs078

7. Falk, Nadja et al. Primary Hyperoxaluria Type 1 with Systemic Calcium

8. Oxalate Deposition: Case Report and Literature Review. Department of Pathology and Laboratory Medicine,University of Texas Health Science Center, Houston, TX. Annals of Clin Lab Scien, vol.43, no. 3, 2013. URL: http://citeseerx.ist.psu.edu/ viewdoc/download?doi=10.1.1.843.904\&rep=rep1\&type=pdf 